\title{
UNUSUAL CASE OF ANAEMIA IN ADULT MALE
}

\begin{tabular}{ll}
$\begin{array}{l}\text { Dr Dnyaneshwar S } \\
\text { Cheke* }\end{array}$ & $\begin{array}{l}\text { Post-graduate resident (Internal medicine) SKNMC \& GH, Pune. } \\
{ }^{*} \text { Corresponding Author }\end{array}$ \\
\hline Dr Isha Desai & Medical officer (internal medicine) DMH, Pune \\
\hline Dr Jitendra Ingole & Professor, MD (Internal Medicine), SKNMC \& GH, Pune \\
\hline \hline
\end{tabular}

ABSTRACT A case of Anaemia which was investigated and found to be a case of Acquired Pure Red Cell Aplasia. Pure red cell aplasia (PRCA) is a syndrome defined by a normocytic normochromic anaemia with severe reticulocytopenia and marked reduction or absence of erythroid precursors from the bone marrow. It is commonly due to primary autoimmune or infection and secondary to myeloproliferative disorder. In this discussion we are presenting a case of normocytic normochromic anaemia which was admitted to our hospital after being investigated and treated several times outside. On extensive workup, we diagnosed the case as PRCA secondary to Thymoma and initiated the appropriate management.

\section{KEYWORDS : Normochromic Normocytic anaemia, Pure Red Cell Aplasia, Thymoma}

A 53 year old male, farmer by occupation, came to the Medicine OPD with the chief complaints of easy fatiguability, generalised weakness and shortness of breath since 1 month. No history relevant to cardiac and respiratory diseases. No history of malena, bleeding tendencies, PR bleed, rash, jaundice night sweat, weight loss and bone pain. He gave a history of hospitalisation for similar complaints two to three times in the past where he was transfused with blood and was started on oral hematenics. He was a Tobacco chewer for 10 years. He had no significant family history. General examination showed significant pallor and no other abnormality. No bruising or petechiae, no platynychia, koilonychia or knuckle pigmentation. Systemic examination revealed no significant finding there were no murmur or organomegaly provisional diagnosis of Outside lab reports showed Hb- $4.8 \mathrm{~g} / \mathrm{dl}$, TLC: 6,400/cu mm, PLT: $1,61,000 / \mathrm{cu} \mathrm{mm}, \mathrm{MCV}-81.3 \mathrm{fl}, \mathrm{MCH}-27.9 \mathrm{pg}$, the peripheral blood smear showed normocytic, normochromic red cells. On admission his labs were $\mathrm{Hb}-5.9 \mathrm{~g} / \mathrm{dl}$, TLC11,770/cumm, Plt-162000/ cu mm, peripheral blood smear Reticulocyte count $-0.6 \%$, Corrected reticulocyte count$0.22 \%$, Serum LDH - $104 \mathrm{u} / \mathrm{L}$, Serum iron- $156 \mathrm{mcg} / \mathrm{dl}$ (50150), TIBC-308mcg/dl(N-300-360ug/dl), S. Ferritin-164ng/ml (N-29-250), random blood sugar-113 mg/dl. Total bilirubin-0.5 mg/dl, Direct bilirubin- $0.3 \mathrm{mg} / \mathrm{dl}$, SGOT -18 u/L, SGPT- $46 \mathrm{u} / \mathrm{L}$, Serum ALP - $75 \mathrm{u} / \mathrm{L}$. Renal function tests and electrolytes were within normal range. USG abdomen showed mild splenomegaly of $14 \mathrm{~cm}$. Bone marrow aspirate showed normocellular to mildly cellular bone marrow with a myeloid : erythroid ratio 5:1. Erythroid series was hypoplastic and shows normoblastic erythropoiesis. Myeloid series - shows progressive maturation. Megakaryocytes - adequate in number suggestive of Normocellular to mildly hypocellular marrow with normoblastic erythropoiesis. Bone marrow biopsy showed normocellular to mildly hypocellular marrow. Myeloid were adequate with all sequential stages of differentiation and maturation. Eosinophils and its precursors were increased. Erythroid series are markedly suppressed. Megakaryocytes were adequate. No evidence of granulomas or any infiltrative pathology. This was suggestive of Acquired red cell aplasia. A Chest X-Ray (figure-1) showed well defined radio opaque shadow measuring $7.5^{*} 2.5 \mathrm{~cm}$ in left hilar region and a $2 \mathrm{~cm}$ well defined oval radio opacity in right upper lung zone in paravertebral region.

\section{Figure 1: Chest X-ray PA view}

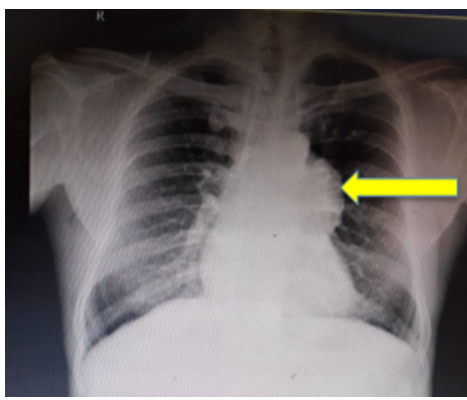

Which was suspected to be due to mass or lymphadenopathy. Simultaneously workup for autoimmune diseases, Parvovirus B19 and Thymoma was done. ANA was negative, dsDNA was negative, Parvovirus B19 antibodies were negative. A HRCT Thorax (figure-2) was s/o suggestive of well defined heterogeneously enhancing soft tissue density lesion in anterior mediastinum in more vascular region likely suggestive of neoplastic origin. Mostly Thymic in origin Lymphoma less likely.

Figure2: HRCT Thorax, showing an anterior mediastinal mass

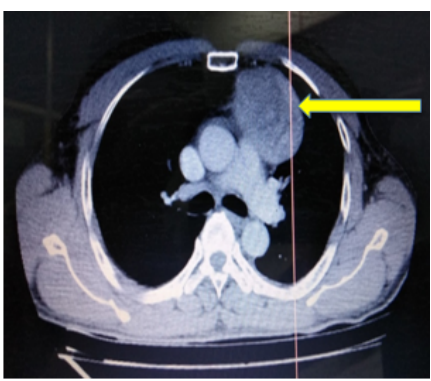

A final diagnosis of Thymoma associated with T-cell suppressing Pure Red cell Aplasia was made. The patient was transfused with 3 units of packed cell volume and was given injectable B complex vitamin and oral hematenics. He was started on steroids with a dose of Prednisolone $60 \mathrm{mg}$ once a day which was then tapered. He was posted for Thymectomy. The patient was operated for thymectomy in same admission and mass of approximately $7 \times 3 \mathrm{~cm}$ was removed (figure-3). After surgery, immunosuppressant therapy was planned after 6 months. 
Figure 3: Excised Thymic Mass

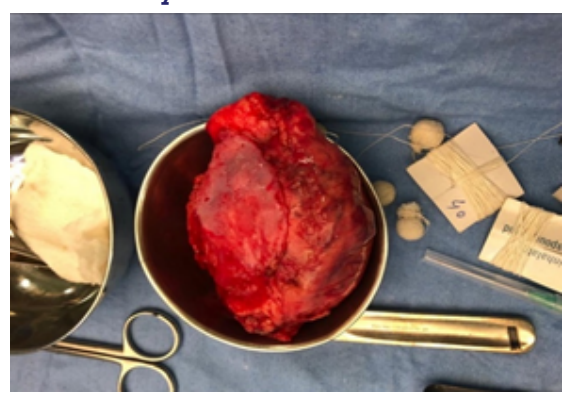

\section{DISCUSSION:}

Pure Red Cell Aplasia is a disorder characterized by normochromic, normocytic anaemia, decreased reticulocyte count, normal counts of other lineages and a normocellular bone marrow with reduced erythroblasts. It was classified as congenital and acquired. Secondary acquired PRCA may be associated with collagen vascular/autoimmune disorders such as systemic lupus erythematosus; lymphoproliferative disorders such as chronic lymphocytic leukaemia or large granular lymphocyte leukaemia; infections, particularly B19 parvovirus; thymoma and other solid tumours; or a variety of other disorders, drugs, or toxic agents. Primary acquired PRCA is an autoimmune disorder in which an immune mechanism interrupts erythroid differentiation. Our case highlights a cause of secondary PRCA due to Thymoma associated with T-cell supressing Pure red cell aplasia. Thymoma is a disorder with the strongest historical association with PRCA. Recent series suggest PRCA is found in less than $5 \%$ of patients with thymoma. The clinical presentation is non-specific and generally presents insidiously, with most individuals lacking signs and symptoms of anaemia until the reduction in hemoglobin and hematocrit becomes quite severe, often to a hematocrit of $<10$ percent. Extreme pallor or decreased exercise tolerance may be the first signs of this disorder in a previously healthy individual. In case of secondary PRCA some symptoms of the associated syndrome may manifest. Evaluation of acquired PRCA should focus on identifying patients with myelodysplastic syndrome presenting with erythroid hypoplasia or PRCA associated with drugs, with B19 parvovirus infection, with thymoma, or with lymphoproliferative disorders, for whom syndrome-specific management is required. The red cells on a peripheral blood count in PRCA are normochromic normocytic. The absolute reticulocyte count is always less than $10,000 / \mu \mathrm{L}$ (reticulocyte percentage, $<1 \%$ ), and in many cases is much lower. The white blood cell count and platelet count are unaffected. Markers of erythropoiesis and ineffective haemolysis are often absent. The diagnosis of PRCA is established when all of the following are present:

I. Normocytic, normochromic anaemia.

II. Absolute reticulocyte count $<10,000 / \mathrm{microL}$ (or reticulocyte percentage $<0.5$ percent; typically $<0.2$ percent).

III. Normal WBC and platelet counts, in the absence of $a$ concurrent disorder such as CLL.

IV. Normocellular bone marrow, with erythroblasts totalling $<1$ percent or proerythroblasts plus basophilic erythroblasts totaling $<5$ percent of nucleated cells.

V. No significant abnormalities in the myeloid, lymphocytic, or megakaryocyte lineages, unless the patient has a concurrent diagnosis of CLL or chronic myeloid leukemia (CML). There may be a slight increase in polyclonal lymphocytes or plasma cells

To establish a diagnosis a bone marrow examination is must. The diagnosis of PRCA is based on the absence or near absence of erythroblasts from an otherwise normal marrow ( $<1 \%$ erythroblasts on the marrow differential count. Large proerythroblasts with vacuolated cytoplasm and pseudopodia ("giant pronormoblasts") are suggestive of B19 parvovirus infection, but are not diagnostic. Iron stains will typically be normal. Because of the paucity of erythroid precursors, ring sideroblasts are difficult to see. If present, ring sideroblasts favor a myelodysplastic syndrome, as would marked hypercellularity. A computed tomography scan of the chest should be performed to rule out a thymoma, which would have potential therapy implications. The goal of treatment is to attain a normal hemoglobin concentration without any requirement for transfusion; a partial response is attainment of transfusion independence with a low but clinically acceptable hemoglobin concentration. The traditional immunosuppression approach began with oral corticosteroids, usually prednisone. Overall, this approach appears to have a $40 \%$ response rate. Prednisone is tapered after a response is obtained, or decreased and used in association with another agent if remission is not obtained. The most effective immunosuppressive agent is cyclosporine. Cyclosporine can be considered the agent of choice for immunosuppression in PRCA, or it can be considered the second-line agent after corticosteroids. A reasonable starting dose is $6 \mathrm{mg} / \mathrm{kg}$ daily. It is sometimes used in association with prednisone $30 \mathrm{mg} / \mathrm{day}$. Cyclosporine trough levels should be monitored with target levels of 150 to $250 \mathrm{ng} / \mathrm{mL}$. Renal and hepatic function should also be monitored. After normalization of the hemoglobin concentration, cyclosporine can be tapered slowly. Maintenance therapy may be required.

Cytotoxic agents such as azathioprine or cyclophosphamide (usually in combination with oral corticosteroids) have been used in patients unresponsive to corticosteroids alone. Rituximab, a humanized anti-CD20 monoclonal antibody, appears to have some efficacy against primary autoimmune PRCA, but is mainly used in PRCA secondary to lymphoproliferative disorders. Antithymocyte globulin, in the doses used for treatment of aplastic anaemia, has a $50 \%$ response rate in primary autoimmune PRCA. Modalities used in refractory primary PRCA with relatively low response rates include intravenous immunoglobulin, plasma exchange, splenectomy, and bone marrow transplantation.

If a thymoma is present in a patient with PRCA, it generally should be resected. Although earlier studies reported more optimistic results, no more than a third of patients will experience remission, and responses may be less than normalization of hemoglobin concentration. Relapses are frequent, and PRCA may develop after resection of a thymoma in patients who did not have PRCA previously. Adjuvant immunosuppressive therapy is typically required. Responses of thymoma-associated PRCA to a combination of immunosuppression and the somatostatin analogue octreotide have been reported. The question of the value of thymectomy in PRCA likely requires re-examination. This will be a challenge requiring a multi-institutional effort, as no single institution is likely to have more than a few cases.

\section{REFERENCES}

1. Means RT Jr. Pure red cell aplasia. Blood 2016; 128:2504

2. Means RT, Dessypris EN, Krantz SB. Treatment of refractory pure red cell aplasia with cyclosporine A: disappearance of IgG inhibitor associated with clinical response. Br J Haematol. 1991;78:114-119

3. Bernard C, Frih H, Pasquet F, et al. Thymoma associated with autoimmune diseases: 85 cases and literature review. Autoimmun Rev. 2016; 15(1):82-92

4. Sawada K, Fujishima N, Hirokawa M Acquired pure red cell aplasia: updated review of treatment. Br J Haematol 2008; 142(4):505-514

5. Dessypris EN. Pure red cell aplasia. Baltimore, MD: Johns Hopkins University Press; 1988 
6. Charles RJ, Sabo KM, Kidd PG, Abkowitz JL. The pathophysiology of pure red cell aplasia: implications for therapy. Blood. 1996;87(11):4831-4838

7. Means RT, Dessypris EN, Krantz SB. Treatment of refractory pure red cell aplasia with cyclosporine A: disappearance of IgG inhibitor associated with clinical response. Br J Haematol. 1991:78:114-119

8. Hirokawa M, Sawada K, Fujishima N, et al; PRCA Collaborative Study Group. Long-term outcome of patients with acquired chronic pure red cell aplasia (PRCA) following immunosuppressive therapy: a final report of the nationwide cohort study in 2004/2006 by the Japan PRCA collaborative study group. Br J Haematol. 2015; 169(6):879-886

9. ThompsonCA, Steensma DP. Pure red cell aplasia associated with thymoma: clinical insights from a 50-year single-institution experience. Br J Haematol. 2006;135(3):405-407

10. Dessypris EN. The biology of pure red cell aplasia. Semin Hematol. $1991 ; 28(4): 275-284$

11. Zaucha R, Zaucha JM, Jassem J. Resolution of thymoma-related pure red cell aplasia after octreotide treatment. Acta Oncol. 2007;46(6):864-865. doi:10.1080/02841860701203578 